\title{
Epitaxial ZnO Films Grown at Low Temperature for Novel Electronic Application
}

\author{
Ł. Wachnicki ${ }^{a, *}$, A. Dużyńska ${ }^{a}$, J.Z. Domagala ${ }^{a}$, B.S. Witkowski ${ }^{a}$, T.A. Krajewski ${ }^{a}$,

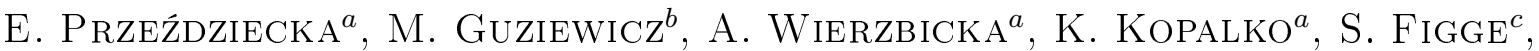 \\ D. Hommel ${ }^{c}$, M. Godlewski ${ }^{a, d}$ AND E. GUZIEWICZ ${ }^{a}$ \\ ${ }^{a}$ Institute of Physics, Polish Academy of Sciences, al. Lotników 32/46, 02-668 Warsaw, Poland \\ ${ }^{b}$ Institute of Electron Technology, al. Lotników 32/46, 02-668 Warsaw, Poland \\ ${ }^{c}$ Institute of Solid State Physics, University of Bremen, Kufsteiner Str. 1, Bremen 28359, Germany \\ ${ }^{d}$ College of Science, Department of Mathematics and Natural Sciences, Cardinal Stefan Wyszynski University \\ Warsaw, Poland
}

\begin{abstract}
Monocrystalline films of zinc oxide were grown at $300^{\circ} \mathrm{C}$ by atomic layer deposition. ZnO layers were grown on various substrates like $\mathrm{ZnO}$ bulk crystal, $\mathrm{GaN}, \mathrm{SiC}$ and $\mathrm{Al}_{2} \mathrm{O}_{3}$. Electrical properties of the films depend on structural quality. Structural quality, surface morphology and optical properties of ZnO films were characterized using X-ray diffraction, scanning electron microscopy, and photoluminescence, respectively. High resolution X-ray diffraction spectra show that the rocking curve FWHM of the symmetrical 00.2 reflection equals to $0.058^{\circ}$ and $0.009^{\circ}$ for $\mathrm{ZnO}$ deposited on a gallium nitride template and a zinc oxide substrate, respectively. In low temperature photoluminescence sharp excitonic lines in the band-edge region with a FWHM equal to $4 \mathrm{meV}, 5 \mathrm{meV}$ and $6 \mathrm{meV}$, for zinc oxide deposited on gallium nitride, zinc oxide and sapphire substrate, respectively.
\end{abstract}

PACS: 81.15.Aa, 61.05.cp, 81.05.Dz

\section{Introduction}

Atomic layer deposition (ALD) introduced by Suntola in 1980 [1] was originally used to obtain monocrystalline films on monocrystalline substrates. At present ALD is widely used to deposit both polycrystalline and monocrystalline layers, including semiconductors and amorphous high- $k$ oxide films $[2,3]$. One of the main advantages of this method is the possibility of low temperature growth. This is also possible on surfaces with highly developed morphology. Characteristic features of the ALD are self limitation and a sequential growth process. This enables the use of very reactive precursors and reduction of a growth temperature, while keeping good crystallographic and optical parameters.

$\mathrm{ZnO}$, which is a II-VI semiconductor with a $3.37 \mathrm{eV}$ direct band gap at room temperature, may be applied in many devices. This includes light emitters, piezoelectric transducers or sensors. $\mathrm{ZnO}$ is also a very prospective material for three-dimensional memories [4] and transparent electronics [5], including the ones with an organic material used as an active part of the device [6-8]. For the latter applications low temperature of $\mathrm{ZnO}$ deposi-

\footnotetext{
* corresponding author; e-mail: lwachn@ifpan.edu.pl
}

tion is essential $[3,6]$ and $300^{\circ} \mathrm{C}$ is an applier limit here. The $\mathrm{ZnO}$ material grown within the above temperature limit is typically polycrystalline. For other applications monocrystallinity of $\mathrm{ZnO}$ films is important. Growth methods that are able to produce such $\mathrm{ZnO}$ films are pulsed laser deposition (PLD), chemical vapor deposition (CVD) with its modifications (like MOCVD) and molecular beam epitaxy (MBE), but temperatures used for monocrystalline $\mathrm{ZnO}$ growth are commonly much higher than $300^{\circ} \mathrm{C}$. They often exceed $600^{\circ} \mathrm{C}$ (for reviews see [10] and references therein). However, already it was often claimed that the ALD method is not suitable for the monocrystalline growth. In a previous paper [6] we reported on a epitaxial $\mathrm{ZnO}$ growth by the ALD, for films grown on a GaN template. This observation motivated the presented study. We demonstrate that crystalline $\mathrm{ZnO}$ can be grown on different substrates like $\mathrm{ZnO}, \mathrm{GaN}$, $\mathrm{SiC}$ and sapphire at restrictive temperature limit. We analyze structural characteristics of these films and relate layer quality with electrical parameters.

\section{Experimental}

$\mathrm{ZnO}$ films studied here were obtained at $300^{\circ} \mathrm{C}$ by the ALD method on $\mathrm{GaN}, \mathrm{SiC}, \mathrm{Al}_{2} \mathrm{O}_{3}$ and $\mathrm{ZnO}$ templates in the Savannah-100 reactor from Cambridge Nanotech. 
We used diethylzinc (DEZn, $\left(\mathrm{C}_{2} \mathrm{H}_{5}\right)_{2} \mathrm{Zn}$ ) as a zinc precursor and deionized water as an oxygen precursor. The zinc oxide film at the surface was created as a product of the double-exchange chemical reaction of DEZn with deionized water

$$
\mathrm{C}_{2} \mathrm{H}_{5}-\mathrm{Zn}-\mathrm{C}_{2} \mathrm{H}_{5}+\mathrm{H}_{2} \mathrm{O} \rightarrow \mathrm{ZnO}+2 \mathrm{C}_{2} \mathrm{H}_{6} .
$$

The structure and the crystallographic orientation of $\mathrm{ZnO}$ layers were determined by X-ray diffraction (XRD). The quality of the layers was investigated by a high resolution X'Pert MRD diffractometer equipped with a X-ray mirror and a two-bounce monochromator at the incident beam. The diffracted beam was measured with a 2-dimensional solid-state X-ray detector - PIXcel. The surface morphology and films cross-section images were obtained by scanning electron microscope (SEM, Hitachi SU-70) with an operation voltage of $15 \mathrm{kV}$. Room temperature photoluminescence (RT PL) studies were performed with a xenon lamp and the CM 2203 spectrometer. Low temperature (LT) PL measurements were obtained with $\mathrm{He}-\mathrm{Cd} 325 \mathrm{~nm}$ laser line using the CCD camera.

\section{Results and discussion}

One micrometer thick films were deposited on substrates with different lattice mismatch to the $\mathrm{ZnO}$ lattice, i.e. gallium nitride template $\left(\mathrm{GaN} / \mathrm{Al}_{2} \mathrm{O}_{3}\right)$, sapphire, silicon carbide and zinc oxide single crystal. The mismatch between $\mathrm{ZnO}$ and $\mathrm{GaN}$ is only $1.9 \%$. Therefore, we expected lower stress and dislocations density. The lattice mismatch for $\mathrm{SiC}$ and $\mathrm{Al}_{2} \mathrm{O}_{3}$ substrates is higher and equals $5.4 \%$ and $31.7 \%$, respectively. In the latter case the actual lattice mismatch is reduced to $18.4 \%$ by rotation of the $\mathrm{ZnO}$ unit cell of growing $\mathrm{ZnO}$ layers with respect to the substrate unit cell by $30^{\circ}$ [11].

The ALD process consists of repeating four deposition steps: deposition of a first precursor; purging the reaction chamber with an inert gas, when non-reacted precursors' and by-products' molecules are removed; deposition of a second precursor; and final purging of the reaction chamber. Thus several parameters affect final quality of $\mathrm{ZnO}$ films. These are lengths of pulses, growth temperature, purging times, etc. Moreover, the crystallographic quality of the ZnO-ALD film as well the surface morphology depends on the kind of precursors used. We found that when using DEZn and water for the $\mathrm{ZnO}$ deposition a long purging time and higher growth temperature are advantageous. The growth with $c$ axis perpendicular to the surface is achieved at these conditions. In this case only the 00.2 peak is observed in the XRD spectrum. In the following experiments we used ALD parameters that are feverous for $\mathrm{ZnO}$ growth with $c$ axis perpendicular to the surface. Deposition temperature was $300^{\circ} \mathrm{C}$. The same conditions were used for the ALD processes in all investigated substrates.

In Fig. 1 SEM images are shown of $\mathrm{ZnO} / \mathrm{SiC}, \mathrm{ZnO} /$ $\mathrm{ZnO}, \mathrm{ZnO} / \mathrm{Si}$ and $\mathrm{ZnO} / \mathrm{GaN}$ interfaces. For the silicon substrate, where the lattice mismatch is very high (about $40 \%$ ) the monocrystalline growth was not achieved. One can see here a columnar growth with a column width about $100 \mathrm{~nm}$. A columnar growth was less evident in case of $\mathrm{ZnO} / \mathrm{GaN}$ and $\mathrm{ZnO} / \mathrm{SiC}$ films, which are of much higher structural quality. We observed that some dislocations coming from the gallium nitride and silicon carbide substrates are spreading into the zinc oxide layer. From the SEM images and epitaxy software calculations (PANAlytical software used for plotting and analyzing rocking curves, 2-axes scans, reciprocal space) one can conclude that zinc oxide films are fully relaxed. An ideal interface and the best $\mathrm{ZnO}$ layer quality are obtained for the homoepitaxial growth i.e., for $\mathrm{ZnO} / \mathrm{ZnO}$ process. In this case the zinc oxide film is fully uniform and neither dislocations nor grain boundaries are seen in the SEM image.

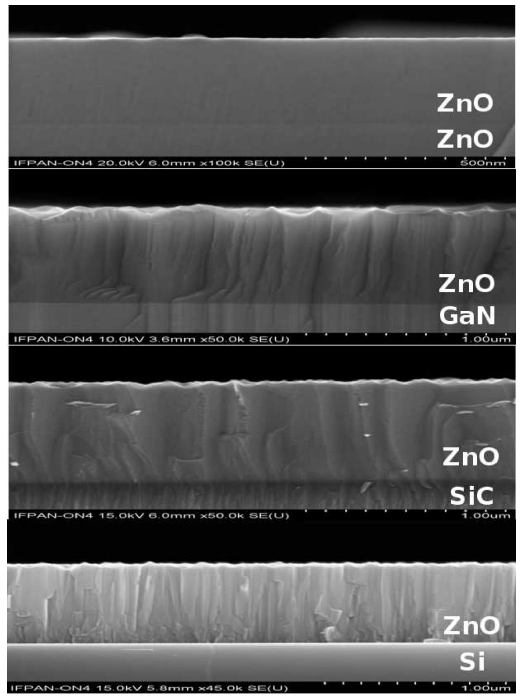

Fig. 1. SEM studies show good structural quality of $\mathrm{ZnO}$ films deposited on different substrates. The best monocrystalline quality was obtained for zinc oxide and gallium nitride substrates.

Figure 2 shows reciprocal space maps (RSM) of the 00.2 reflection from the epitaxial $\mathrm{ZnO}$ films deposited on $\mathrm{GaN}, \mathrm{SiC}, \mathrm{Al}_{2} \mathrm{O}_{3}$ and $\mathrm{ZnO}$. The RSM maps confirm that $\mathrm{ZnO}$ layers obtained are monocrystalline, with a full width at half maximum (FWHM) of the rocking curve (00.2 reflection) equal to $0.009^{\circ}$ and $0.058^{\circ}$ for $\mathrm{ZnO}$ $\mathrm{ZnO}$ and $\mathrm{ZnO} / \mathrm{GaN}$, respectively. The reason of rather high diffuse scattering (elliptical shape isocontures with 3 ordered smaller intensity than for the Bragg peak) is different for these two substrates. Films grown on GaN templates reflect the structural imperfection of the templates ( $\mathrm{GaN} /$ sapphire; dislocation density in order of the $\left.10^{9} \mathrm{~cm}^{-2}\right)$. Ellipses visible in Fig. $2 \mathrm{~b}$ are twice more elongated in $Q_{x}$ direction than for the $\mathrm{ZnO} / \mathrm{ZnO}$ case (Fig. 2c). We suppose that diffuse scattering for homoepitaxial growth of $\mathrm{ZnO}$ is correlated with the surface imperfection. For $\mathrm{ZnO} / \mathrm{Al}_{2} \mathrm{O}_{3}$ and $\mathrm{ZnO} / \mathrm{SiC}$ the $\mathrm{FWHM}$ 

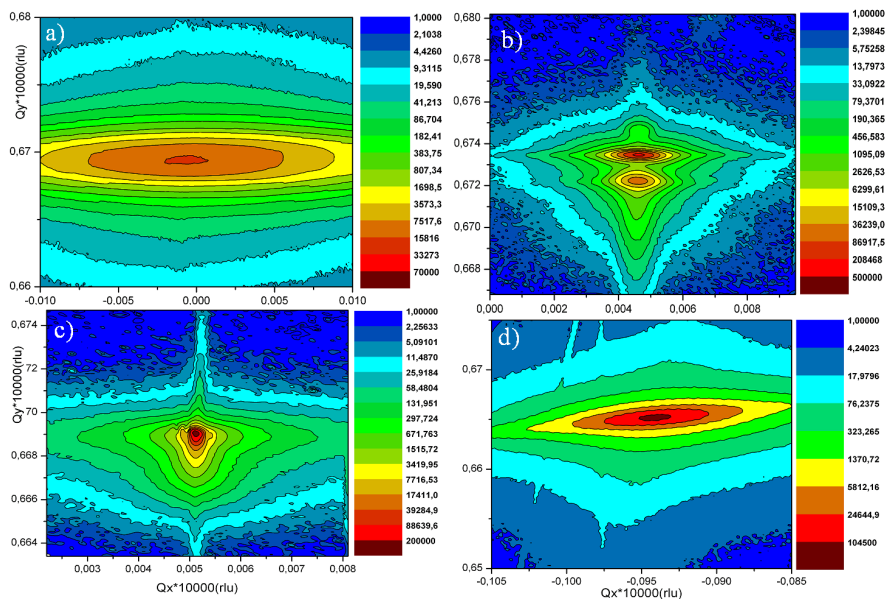

Fig. 2. Reciprocal space maps of the 00.2 reflection from zinc oxide deposited on (a) sapphire, (b) gallium nitride, (c) zinc oxide bulk crystal, (d) silicon carbide.

of the rocking curve is equal to $0.991^{\circ}$ and $0.345^{\circ}$, respectively.

The lattice parameters of the obtained films were determined from the RSMs of symmetrical 00.2 and the asymmetrical $-1-1.4$ reflections. In Table we present the lattice parameters of $\mathrm{ZnO}$. The data for films deposited on different substrates are compared with single crystal lattice parameters of $\mathrm{ZnO}$ [12]. One can see that lattice constants of ZnO-ALD films are very similar to these of a single $\mathrm{ZnO}$ crystal. For $\mathrm{ZnO} / \mathrm{ZnO}$ homoepitaxial films the differences between lattice parameters of bulk and epitaxial $\mathrm{ZnO}$ are within the error limit. Surprisingly, the values of lattice parameters for the $\mathrm{ZnO}$ layer grown on sapphire are only about $0.02 \%$ larger than these of a $\mathrm{ZnO}$ single crystal. However, the higher FWHM of the 00.2 peak for $\mathrm{ZnO}$ films deposited on $\mathrm{Al}_{2} \mathrm{O}_{3}$ suggests a higher screw dislocation density. The largest differences in lattice parameters are noticed for $\mathrm{ZnO}$ films deposited on $\mathrm{GaN}$ and $\mathrm{SiC}$ substrates. For $\mathrm{ZnO} / \mathrm{GaN} a$ and $c$ lattice constants are $1.1 \%$ larger and $1.6 \%$ smaller than lattice constants of a zinc oxide single crystal. This shows that the layer deposited on gallium nitride is tensile strained in the (0001) plane. A similar situation is observed for the $\mathrm{ZnO} / \mathrm{SiC}$ film. The optical properties of epitaxial $\mathrm{ZnO}$ films were characterized by RT and LT PL.

Figure 3 shows RT PL spectra of zinc oxide deposited on $\mathrm{Al}_{2} \mathrm{O}_{3}, \mathrm{GaN}$ and $\mathrm{ZnO}$ single crystal. We observed here high edge luminescence in the blue spectral region. Defect-related PL usually observed as a broad band in the visible spectral region is not present in any measured films. The spectral position of the edge emission is similar for all investigated $\mathrm{ZnO}$ layers and is $3.25 \mathrm{eV}$. The strongest edge luminescence is observed for $\mathrm{ZnO}$ films grown on a sapphire substrate. It is probably related to the full relaxation of $\mathrm{ZnO} / \mathrm{Al}_{2} \mathrm{O}_{3}$ films which was postulated above. PL spectra measured at $12 \mathrm{~K}$ are presented in Fig. 4. PL shows sharp excitonic luminescence with

\section{TABLE}

Lattice constants parameters for zinc oxide thin films deposited on zinc oxide single crystal, gallium nitride, sapphire, silicon carbide. The lattice constant of zinc oxide single crystal are $a=3.2495 \AA$ and $c=5.2069 \AA[9]$.

\begin{tabular}{c|c|c}
\hline \hline Substrates & Lattice $a[\AA]$ & Lattice $c[\AA]$ \\
\hline $\mathrm{GaN}$ & 3.2534 & 5.1986 \\
$\mathrm{SiC}(4 \mathrm{H})$ & 3.2513 & 5.1957 \\
$\mathrm{Al}_{2} \mathrm{O}_{3}$ & 3.2488 & 5.2057 \\
$\mathrm{ZnO}$ & 3.2492 & 5.2067
\end{tabular}

FWHM values of $4 \mathrm{meV}, 5 \mathrm{meV}$ and $6 \mathrm{meV}$ for $\mathrm{ZnO} / \mathrm{GaN}$, $\mathrm{ZnO} / \mathrm{ZnO}$ and $\mathrm{ZnO} / \mathrm{Al}_{2} \mathrm{O}_{3}$, respectively. In PL spectrum of zinc oxide deposited on sapphire we observe two peaks: higher one at $3.36 \mathrm{eV}$ corresponding to neutral donor-bound exciton recombination [13], and a smaller peak at $3.33 \mathrm{eV}$ likely also corresponding to a donor-bound exciton transition [14]. The same peaks are seen for zinc oxide deposited on the gallium nitride substrate, but with different intensities. Donor-bound excitonic emission at $3.33 \mathrm{eV}$ is dominant in the $\mathrm{ZnO} / \mathrm{GaN}$ spectrum. For $\mathrm{ZnO}$ obtained on gallium nitride we notice phonon replied of the PL peaks. This confirms the good crystallographic quality of the films studied. Homoepitaxial zinc oxide layers show much weaker low-temperature photoluminescence. We attribute this to a diffusion of lithium and/or potassium contaminations from the commercial zinc oxide substrate to $\mathrm{ZnO}$ layers. Our films were grown on substrates obtained by hydrothermal method which contain large $\mathrm{Li}$ and $\mathrm{K}$ concentration.

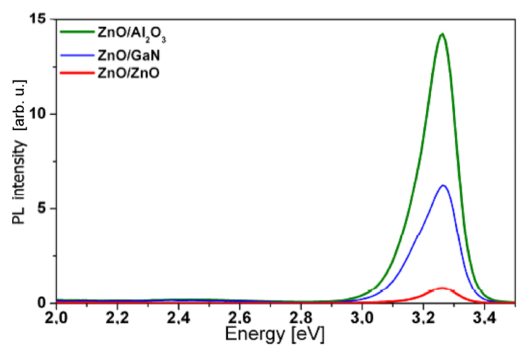

Fig. 3. Room temperature photoluminescence for zinc oxide thin films deposited on gallium nitride, single crystal zinc oxide and sapphire.

We note here that structural and optical parameters shown above are comparable with the ones reported for epitaxial $\mathrm{ZnO}$ films obtained at much higher temperatures by CVD or MBE [15]. For example, $\mathrm{ZnO}$ grown on GaN substrate by CVD at $700^{\circ} \mathrm{C}$ shows the rocking curve of 1000 arcsec [15], which is much higher than for our $\mathrm{ZnO} / \mathrm{GaN}$ films (250 arcsec).

The electrical parameters of $\mathrm{ZnO} / \mathrm{GaN}$ and $\mathrm{ZnO} /$ $\mathrm{Al}_{2} \mathrm{O}_{3}$ have been examined by the Hall effect measurements. These investigations were performed at room 


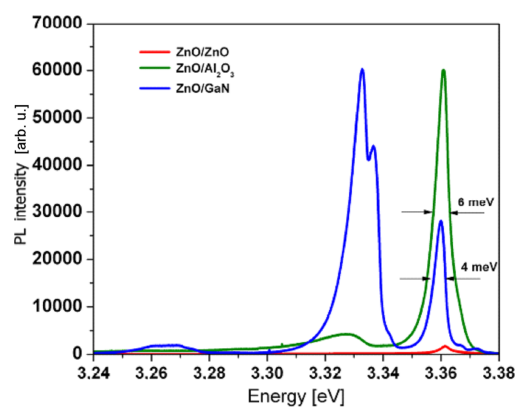

Fig. 4. Low temperature photoluminescence for zinc oxide thin films deposited on gallium nitride substrate, single crystal zinc oxide and sapphire.

temperature in the van der Pauw configuration using the RH2035 PhysTech GmbH system equipped with a permanent magnet giving a field of $0.426 \mathrm{~T}$. The free carrier concentration was $1.8 \times 10^{18} \mathrm{~cm}^{-3}$ for a $\mathrm{ZnO} / \mathrm{GaN}$ film and $2.2 \times 10^{18} \mathrm{~cm}^{-3}$ for a $\mathrm{ZnO} / \mathrm{Al}_{2} \mathrm{O}_{3}$ layer. These values are about one order of magnitude lower than obtained for a $\mathrm{ZnO}$ film deposited on a glass substrate in the same ALD process, where the ZnO layer was polycrystalline. For $\mathrm{ZnO} /$ glass films we obtained $n$ concentration of $1.3 \times 10^{19} \mathrm{~cm}^{-3}$. The measured mobility of carriers was higher for epitaxial layers than for the polycrystalline films. The mobility is also strongly correlated with the structural quality of the film. The mobility $\mu$ for $\mathrm{ZnO} / \mathrm{GaN}$ film (the FWHM of the rocking curve 250 arcsec) was $167 \mathrm{~cm}^{2} /(\mathrm{Vs})$, whereas $\mu$ for $\mathrm{ZnO} / \mathrm{Al}_{2} \mathrm{O}_{3}$ layer (the FWHM of the rocking curve 3560 arcsec) equals $39 \mathrm{~cm}^{2} /(\mathrm{V} \mathrm{s})$. For comparison, the mobility of carriers in the reference polycrystalline $\mathrm{ZnO} /$ glass film is about $30 \mathrm{~cm}^{2} /(\mathrm{V} \mathrm{s})$.

\section{Conclusions}

In conclusion, we obtained monocrystalline $\mathrm{ZnO}$ films on $\mathrm{GaN}$ and bulk $\mathrm{ZnO}$ substrates by atomic layer deposition at temperature $300^{\circ} \mathrm{C}$. The structural and optical parameters "as-grown" $\mathrm{ZnO}$ films are surprisingly good despite low growth temperature. We note here that structural and optical parameters shown above are comparable with the ones reported for epitaxial $\mathrm{ZnO}$ films obtained at much higher temperatures by CVD or MBE. For example, $\mathrm{ZnO}$ grown on GaN substrate by CVD at $700^{\circ} \mathrm{C}$ shows the rocking curve of 1000 arcsec, which is much higher than for our $\mathrm{ZnO} / \mathrm{GaN}$ films (250 arcsec). Electrical properties of epitaxial $\mathrm{ZnO}$ films strongly correlate with the FWHM of the rocking curve. For $\mathrm{ZnO} /$ GaN films mobility of carriers is a $169 \mathrm{~cm}^{2} /(\mathrm{V} \mathrm{s})$ and free electron concentration at RT is $1.8 \times 10^{18} \mathrm{~cm}^{-3}$. All these parameters favorably compare with the ones obtained at higher temperature with MBE or MOCVD.

\section{Acknowledgments}

The research was partially supported by: European Union within European Regional Development Fund, through grant Innovative Economy (POIG.01.01.02-00$-008 / 08$ ) and by grant no. 0663/B/T02/2008/35 from the Polish Ministry of Science and Higher Education.

\section{References}

[1] T. Suntola, in: Growth Mechanisms and Dynamics, Ed. D.T.J. Hurle, Handbook of Crystal Growth, Vol. 3, Elsevier, Amsterdam 1994, p. 601.

[2] I.A. Kowalik, E. Guziewicz, K. Kopalko, S. Yatsunenko, A. Wójcik-Głodowska, M. Godlewski, P. Dużewski, E. Łusakowska, W. Paszkowicz, J. Cryst. Growth 311, 1096 (2009).

[3] E. Guziewicz, I.A. Kowalik, M. Godlewski, K. Kopalko, V. Osinniy, A. Wójcik, S. Yatsunenko, E. Łusakowska, W. Paszkowicz, M. Guziewicz, J. Appl. Phys. 103, 033515 (2008).

[4] E. Guziewicz, M. Godlewski, T.A. Krajewski, Ł. Wachnicki, G. Łuka, J.Z. Domagała, W. Paszkowicz, B.J. Kowalski, B.S. Witkowski, A. Duzynska, A. Suchocki, Phys. Status Solidi B 247, 1611 (2010).

[5] R.L. Hoffman, B.J. Norris, J.F. Wager, Appl. Phys. Lett. 82, 733 (2003)

[6] Ł. Wachnicki, T. Krajewski, G. Łuka, B. Witkowski, B. Kowalski, K. Kopalko, J.Z. Domagala, M. Guziewicz, M. Godlewski, E. Guziewicz, Thin Solid Films 518, 4556 (2010).

[7] M. Godlewski, E. Guziewicz, J. Szade, A. Wójcik-Godowska, Ł. Wachnicki, T. Krajewski, K. Kopalko, R. Jakieła, S. Yatsunenko, E. Przezdziecka, P. Kruszewski, N. Huby, G. Tallarida, S. Ferrari, Microelectron. Eng. 85, 2434 (2008).

[8] T. Krajewski, E. Guziewicz, M. Godlewski, Ł. Wachnicki, I.A. Kowalik, A. Wojcik-Glodowska, M. Łukasiewicz, K. Kopalko, V. Osinniy, M. Guziewicz, Microelectron. J. 40, 293 (2009).

[9] E. Guziewicz, M. Godlewski, T. Krajewski, Ł. Wachnicki, A. Szczepanik, K. Kopalko, A. Wójcik-Godowska, E. Przeździecka, W. Paszkowicz, E. Łusakowska, P. Kruszewski, N. Huby, G. Tallarida, S. Ferrari, J. Appl. Phys. 105, 1 (2009).

[10] R. Triboulet, J. Perrière, Prog. Cryst. Growth Charact. Mater. 47, 65 (2003).

[11] H. Morkoç, Ü. Özgur, in: Zinc Oxide, Wiley-VCH, Weinheim 2009, p. 89.

[12] C. Klingshirn, Phys. Status Solidi B 244, 3027 (2007).

[13] B.K. Meyer, J. Sann, D.M. Hofmann, C. Neumann, A. Zeuner, Semicond. Sci. Technol. 20, S62 (2005).

[14] E. Przezdziecka, T. Krajewski, L. Wachnicki, A. Szczepanik, A. Wójcik-Godowska, S. Yatsunenko, E. Lusakowska, W. Paszkowicz, E. Guziewicz, M. Godlewski, Acta Phys. Pol. A 114, 1303 (2008).

[15] S. Lautenschlaeger, S. Eisermann, M.N. Hofmann, U. Roemer, M. Pinnisch, A. Laufer, B.K. Meyer, H. von Wenckstern, A. Lajn, F. Schmidt, M. Grundmann, J. Blaesing, A. Krost, J. Cryst. Growth 312, 2078 (2010). 